\title{
MOSFET Two-Dimensional Doping Profile Determination
}

\author{
N. Khalil and J. Faricelli \\ Advanced Semiconductor Development, Digital Equipment Corporation \\ Hudson, MA 01749, USA
}

\begin{abstract}
Direct experimental measurement techniques have had limited success in the determination of the two-dimensional (2D) doping profile of a MOSFET. In this paper, we describe an alternative methodology that uses source/drain (S/D) diode and gate overlap capacitance measurements to determine the $2 \mathrm{D}$ profile by inverse modeling [1]. Our approach is based on the optimized tensor product spline (TPS) representation of the profile. We use nonlinear multiple outputs, least squares optimization to extract the values of the B-splines coefficients.
\end{abstract}

\section{Introduction}

The determination of the two-dimensional (2D) doping profile of a MOSFET is a crucial factor for device characterization and a very important input for device simulation. Direct experimental measurements of $2 \mathrm{D}$ doping profiles have met with limited success on very shallow junction devices, or rely on heroic experimental preparation techniques that require sophisticated data reduction or complicated angle lap and stain techniques $[2,3,4]$. In this paper, we describe a method for determining the 2D profile from MOSFET and S/D diode electrical measurements by inverse modeling [1]. We rely mainly on measuring various capacitances under different bias conditions that "probe" various portions of the doping profile by depleting or accumulating that region of carriers. In addition, we use one-dimensional profiling information from secondary ion mass spectroscopy (SIMS) or deep depletion $\mathrm{C}-\mathrm{V}$ measurements as the "boundary conditions" of our extracted $2 \mathrm{D}$ profile. An initial guess is derived from these $1 \mathrm{D}$ measurements. We then perform an optimization using device simulation to reproduce the measurements numerically, and adjust the doping to minimize the difference between measurement and simulation.

\section{Profile Representation}

We parameterize 2D doping profiles by representing their logarithm as a tensor product spline (TPS) [5]. This representation greatly decreases the amount of computation as compared to the hundreds of parameters required by a straightforward 
mesh representation. It is more general than other analytically based approximation functions. Moreover, the local approximation properties of the B-splines makes it suitable for the extraction procedure. Using the TPS representation a $2 \mathrm{D}$ doping profile is defined by its knots sequences $\mathbf{t}_{\mathbf{x}}$ and $\mathbf{t}_{\mathbf{y}}$ and the values of its coefficients $\mathbf{c}_{\mathrm{ij}}$ and is given by:

$$
f(x, y)=\sum_{i=1}^{n x} \sum_{j=1}^{n y} \mathbf{c}_{\mathbf{i j}} * B_{i, k_{x}, t_{x}}(x) * B_{j, k_{y}, t_{y}}(y)
$$

Where $B_{i, k, t}$ is the ith B-spline of order $k$ for the knot sequence $\mathbf{t}, n x$ and $n y$ the number of knots in the $\mathrm{X}$ and $\mathrm{Y}$ direction respectively.

We represent the donors and the acceptors in a device by two TPS with a common $\mathbf{t}_{\mathbf{x}}$ sequence but different $\mathbf{t}_{\mathbf{y}}$ sequences. For fixed orders $k_{x}, k_{y}$, and $\mathbf{t}_{\mathbf{y}}$ sequences, we can write the net doping as:

$$
\operatorname{net}(x, y)=F\left(\mathbf{t}_{\mathbf{x}}, \alpha_{\mathbf{i j}}, \delta_{\mathbf{i j}}\right)
$$

Where $\alpha_{\mathrm{ij}}$ and $\delta_{\mathrm{ij}}$ are the splines coefficients for the acceptors and donors.

\section{2D Extraction}

We use three different capacitances in our extraction: The gate to S/D overlap capacitance $C_{o v}$, and the depletion capacitance per unit area $C a$, and per unit perimeter $C_{p}$ of the reverse $\mathrm{S} / \mathrm{D}$ junction diode. We calculate the capacitances by integrating the appropriate MINIMOS [6] charges. We avoid numerical problems by resorting to grid refinement and careful handling of rounding and integration errors. As done experimentally, we determine the values of $C_{a}$ and $C_{p}$ from the $\mathrm{S} / \mathrm{D}$ diode capacitance values of two devices with different $S / D$ area sizes.

The capacitances are a nonlinear function of the net doping. Using (2) we can write this functional relationship as:

$$
\mathbf{C}=F\left(\mathbf{V}, \mathbf{t}_{\mathbf{x}}, \alpha_{\mathbf{i j}}, \delta_{\mathbf{i j}}\right)
$$

Where $\mathbf{C}$ is the vector of capacitance values: $C_{a}, C_{p}$, and $C_{o v}$; and $\mathbf{V}$ is the input vector of the device bias voltages.

The extraction problem reduces to determining the values of the $\mathbf{t}_{\mathbf{x}}$ sequence and the sets of coefficients $\alpha_{\mathbf{i j}}$ and $\delta_{\mathbf{i j}}$. We use a nonlinear least square, multiple outputs, optimizer based on the well-known Levenberg-Marquardt algorithm. The Jacobian is calculated by finite differences with usage of the Broyden update scheme [7] to reduce the computational load. The overall figure of merit of the optimization can be formulated as follows:

$$
\sum_{i=1}^{m}\left(\frac{C_{a}^{e x p}-C_{a}^{s i m}}{C_{a}^{e x p}}\right)^{2}+\left(\frac{C_{p}^{e x p}-C_{p}^{s i m}}{C_{p}^{e x p}}\right)^{2}+\left(\frac{C_{o v}^{e x p}-C_{o v}^{s i m}}{C_{o v}^{e x p}}\right)^{2}
$$

Starting from $1 D$ profile information for the channel and the S/D regions, we perform a curve fitting optimization to generate their $1 \mathrm{D}$ spline representations. We assume the $1 \mathrm{D}$ channel profile is spread uniformly in the horizontal dimension and the $\mathrm{S} / \mathrm{D}$ profile to have a certain lateral subdifusion, and we generate the initial TPS representations accordingly.

We then extract from the capacitance data values for the the $\mathbf{t}_{\mathbf{x}}$ knots sequence, and the coefficients $\delta_{\mathrm{ij}}$ and $\alpha_{\mathrm{ij}}$. The extraction is done in multiple iterations using different sets of capacitances data. To avoid redundancy, we only optimize the coefficients that are of the same sign as the net doping at the knot location. 


\section{Results}

To validate the proposed extraction methodology, we show the results of of applying our extraction to two examples. In example 1, the original profile consisted of two TPS profiles for donors and acceptors. We also assumed the knot locations to be known. For example 2, the original profile was formed by rotating $1 \mathrm{D}$ profiles for both donors and acceptors.

As shown in figures 1 and 2, we obtain good agreement between the original and extracted lateral surface doping. This is due to the strong dependence of the gate overlap data in accumulation on the surface doping. However the resolution of the method deteriorates as the distance from the surface increases. This is apparent in figures 3 and 4 where the lateral doping at $0.1 \mu$ in depth is plotted. For both examples, figures 5 and 6 show that the spatial delineation of the $2 \mathrm{D}$ junction is good. As expected, the accuracy of the extraction is higher in example 1 where there are no errors due to knots placement and 1D spline approximation.

\section{Conclusion}

We have presented a 2D MOSFET profiling method based on inverse modeling of capacitance data. The method is non-destructive and provide important information for device characterization. We plan on further improving the method by using MOSFET threshold and drain current data in our extraction.

\section{References}

[1] G. J. L. Ouwerling, Nondestructive One-And Two-Dimensional Doping Profiling By Inverse Methods, Delft University of Technology, UMI, Ann Arbor MI, 1989.

[2] S. H. Goodwin-Johansson, R. Subrahmanyan , C. E. Floyd and H. Z. Massoud, "Two-Dimensional impurity profiling with emission computed tomography techniques", IEEE-TCAD, CAD-8(4),1989.

[3] R. Subrahmanyan, H. Z. Massoud and R. B. Fair, "Experimental characterization of two-dimensional dopant profiles in silicon using chemical staining", Applied Physics Letters, 52(25), 1988.

[4] S. Kordic, E. Van Leonen, D. Dijkkamp, A. Hoeven and H. Moraal, "Scanning Tunneling Microscopy on Cleaved Silicon PN Junctions", IEDM Technical Digest, 1989, pp.277-280.

[5] Carl De Boor, A Practical Guide to Splines , Springer-Verlag New York Inc., 1978.

[6] S. Selberherr, A. Schultz and H. W. Protzl, "MINIMOS - A Two-Dimensional MOS Transistor Analyser", IEEE Transactions on Electron Devices, ED-27 (8), August 1980.

[7] C. G. Broyden, in Numerical Methods for Unconstrained Optimization, W. Murray, Ed., Academic Press, 1972. 
Figure 1: Lateral Surface Doping (EX. 1)

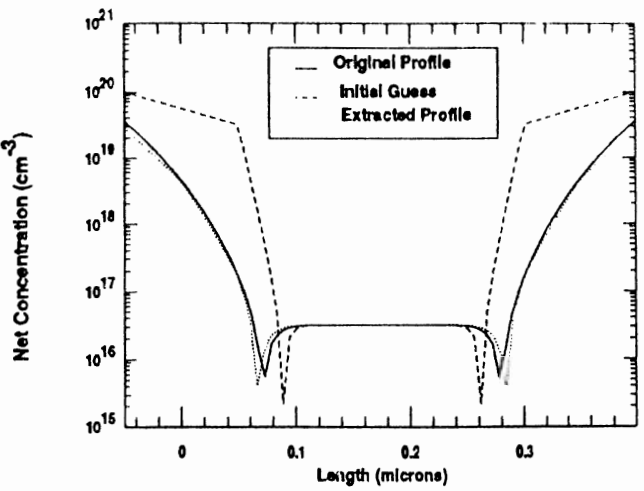

Figure 3: Doping at $0.1 \mu$ Depth (EX. 1)

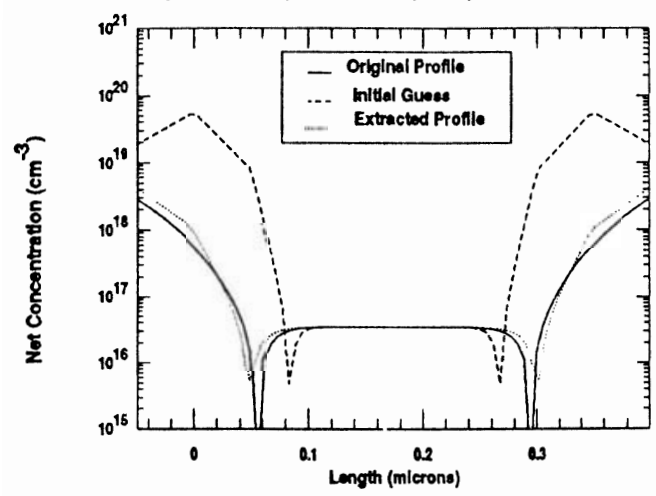

Figure 5: Junctions Comparison (EX. 1)

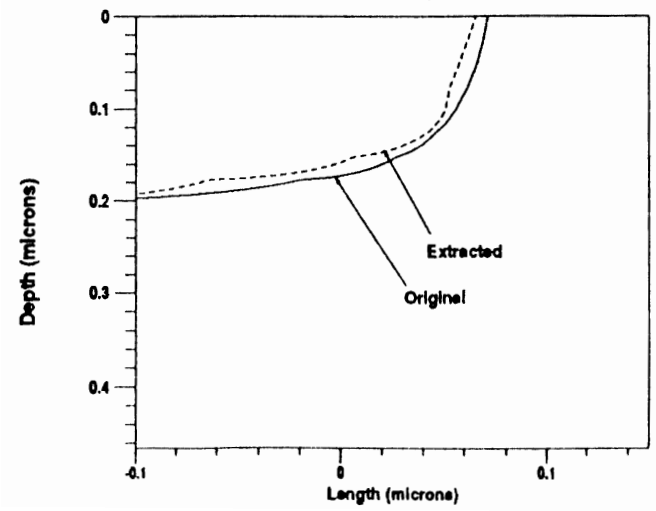

Figure 2: Lateral Surface Doping (EX. 2)

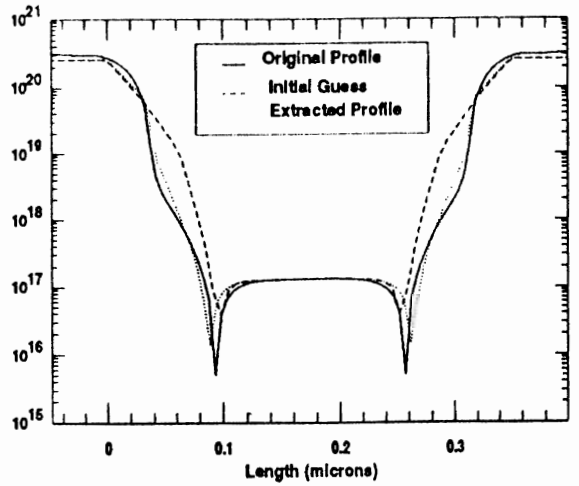

Figure 4: Lateral Doping at $0.1 \mu$ Depth (EX. 2)

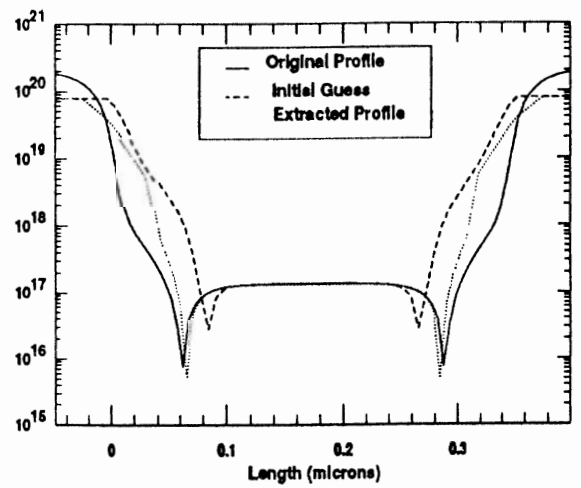

Figure 6: Junctions Comparison (EX. 2)

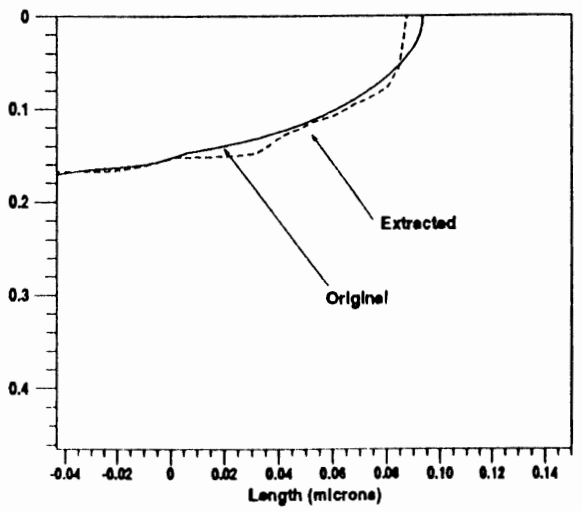

